\title{
Computational modeling of angiogenesis: towards a multi-scale understanding of cell-cell and cell-matrix interactions
}

Sonja E.M. Boas*, Margriet M. Palm*, Pieter Koolwijk and Roeland M.H. Merks

\begin{abstract}
Combined with in vitro and in vivo experiments, mathematical and computational modeling are key to unraveling how mechanical and chemical signaling by endothelial cells coordinates their organization into capillary-like tubes. While in vitro and in vivo experiments can unveil the effects of for example environmental changes or gene knockouts, computational models provide a way to formalize and understand the mechanisms underlying these observations. This chapter reviews recent computational approaches to model angiogenesis, and discusses the insights they provide in the mechanisms of angiogenesis.

We introduce a new cell-based computational model of an in vitro assay of angiogenic sprouting from endothelial monolayers in fibrin matrices. Endothelial cells are modeled by the Cellular Potts Model, combined with continuum descriptions to model haptotaxis and proteolysis of the extracellular matrix. The computational model demonstrates how a variety of cellular structural properties and behaviors determine the dynamics of tube formation. We aim to extend this model to a multi-
\end{abstract}

* these authors contributed equally to this work

Sonja E.M. Boas

Centrum Wiskunde \& Informatica, 1098 XG Amsterdam, The Netherlands; Netherlands Consortium for Systems Biology/Netherlands Institute for Systems Biology, 1098 XG Amsterdam, The Netherlands, e-mail: S.E.M.Boas@ @wi.nl

Margriet M. Palm

Centrum Wiskunde \& Informatica, 1098 XG Amsterdam, The Netherlands; Netherlands Consortium for Systems Biology/Netherlands Institute for Systems Biology, 1098 XG Amsterdam, The Netherlands, e-mail: M.M.Palm@cwi.nl

Pieter Koolwijk

VU University Medical Center, Van der Boechorststraat 7 Amsterdam, e-mail: P.Koolwijk@vumc.nl

Roeland M.H. Merks

Centrum Wiskunde \& Informatica, 1098 XG Amsterdam, The Netherlands; Netherlands Consortium for Systems Biology/Netherlands Institute for Systems Biology, 1098 XG Amsterdam, The Netherlands, e-mail: R.M.H.Merks@cwi.nl 
scale model in the sense that cells, extracellular matrix and cell-regulation are described at different levels of detail and feedback on each other. Finally we discuss how computational modeling, combined with in vitro and in vivo modeling steers experiments, and how it generates new experimental hypotheses and insights on the mechanics of angiogenesis.

\section{Introduction}

Blood vessel growth is essential during embryogenesis, but is also a prominent aspect of diseases such as cancer, rheumatoid arthritis and retinopathy. Angiogenesis research can benefit from computational models in three ways. Firstly, computational models help to gain an overview in this complex system by testing which components and interactions are minimally required. These components and interactions can then be examined to understand their function and predict their effects. Computational models are therefore not only useful to gain mechanistic understanding of angiogenesis, but also to find new therapeutic targets. Secondly, computational models can discriminate between and select from alternative hypotheses. Often, more than one hypothesis explains a biological observation, such as network formation from dispersed endothelial cells. Computational models can test the sufficiency of each hypotheses to reproduce the biological observations. Predictions that result from these models can be validated experimentally to support or reject the tested hypotheses. Thirdly, computational models can connect and combine knowledge on single proteins and mechanisms to examine angiogenesis as a system. Experimental research is often limited to a specific step or protein in angiogenesis and does not grasp how this part is integrated in the whole. Ultimately, computational models include processes at multiple scales, like extracellular matrix, cells, and cell-regulation simultaneously. Such multi-scale models are the next step in computational modeling to make the transition to angiogenesis in the body.

In the first section, computational models of network formation and sprouting are reviewed. These models address questions that have been raised by experimental observations and thereby give new insights in angiogenesis. It concludes by discussing the current state of multi-scale modeling. The next section gives a practical example of how computational models can be used in angiogenesis research and shows how systems biology, a continuous cooperation between computational and experimental biologists, drives development of computational models. To do so, we introduce a new computational model of sprouting, based on an experimental model of capillary-like tube formation by Koolwijk et al. [19]. Finally we will discuss which steps should be taken in angiogenesis research to further evolve computational modeling. 


\section{Computational models of angiogenesis}

The first models of angiogenesis were continuum models that describe angiogenesis in terms of the spatial density of cells $[2,22,31,36]$. The main advantage of these models is that they can often be solved analytically, but they are often too abstract to mimic angiogenesis realistically. More complex techniques allow for a more detailed description of angiogenesis, which yields more realistic models. Such techniques include discrete methods such as particle based modeling that describe cells as point-like particles [3, 42] and cell-based models [16, 41, 40] that also explicitly model the cell shape and membrane. These discrete methods are often combined with continuum models, creating a hybrid model $[4,6,24,29]$ in order to utilize the strength of both methods.

This section reviews computational models of angiogenic network formation and sprouting. Network formation involves the collective behavior of cells and the interaction of cells with their environment. Models of sprouting angiogenesis are used to describe angiogenesis induced by cells in hypoxic tissues, e.g, a tumor.

\subsection{Network formation}

During early vascular development endothelial cells join into a primitive vascular network. Vascular network formation can be mimicked in vitro by seeding endothelial cells on a suitable matrix containing nutrients and angiogenic factors; for example Figure 1a shows endothelial cells seeded on Matrigel matrix forming a networklike pattern. The conditions in in vitro network formation experiments differ greatly from in vivo angiogenesis. Yet, specific cases of angiogenesis result in similar vascular networks such as angiogenesis in the yolk sac and retinal angiogenesis. In both cases the vasculature arises from a vascular plexus containing endothelial cells.

In vitro experiments showed that, after the network is formed, almost all matrix is located beneath the cells [46]. This led to the hypothesis that cells pull on the matrix, resulting in matrix accumulation below cell clusters. The pulling forces of the cells also cause the formation of tension lines, radiating from the clusters, in the surrounding matrix, along which cells migrate [22]. This model assumes that cells can exert traction on the matrix, which results in matrix deformation and heterogeneity of strain in the matrix. Cells preferentially move along the orientation of high stress. The model suggests that matrix remodeling suffices for network formation.

Namy and coworkers combine the effects of cell traction with haptotactic cell migration along matrix gradients [31] (Figure 1b). They founds an optimal cell density at which networks can be created, corresponding with experimental observations [44]. Similarly, a range of matrix stiffness, which is linked to the fibrin density of the experimental matrix, was tested. This model suggested that active cell migration may be required for network formation which contradicts the observations by Manoussaki et al. [22]. 


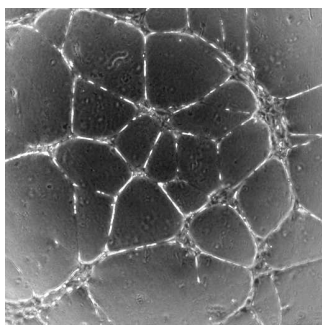

(a)

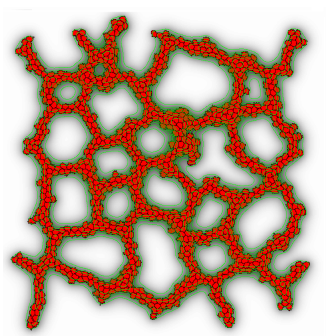

(d)

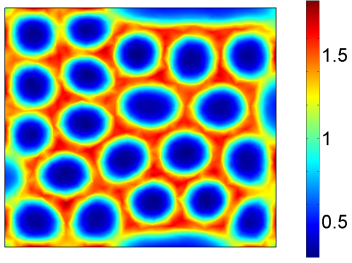

(b)

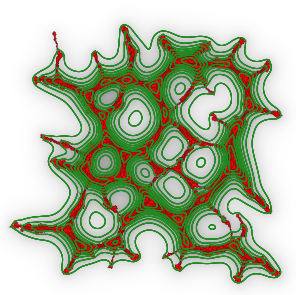

(e)

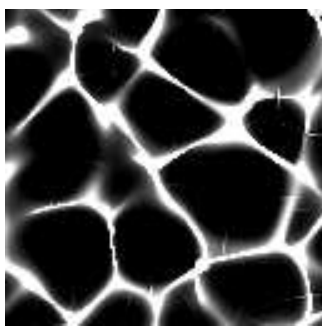

(c)

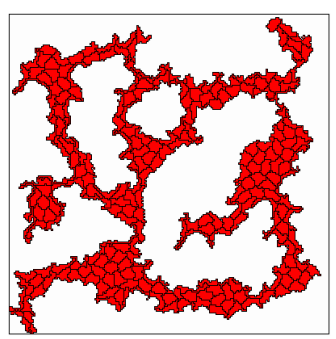

(f)

Fig. 1: Overview of vascular network formation. (a) shows a vasculature grown in vitro with HUVEC on Matrigel. (b) illustrates the networks formed with the mechanical continuum model [31]. (c) shows the outcome of the chemical continuum model [36]. (d) and (e) show the networks formed with the chemical cell-based model, respectively with contact inhibition (d) [29] or cell elongation (e) [24]. (f) illustrates the networks formed with the cell-based model with preferential attraction to elongated structures [41]. All images were reproduced with the publishers permission.

Both previous models consider mechanical interactions between cells and the matrix to be the driving forces for network formation. Serini et al. [11, 36] proposed that chemotaxis is the driving force of network formation [36]. In the in vitro models cells move predominantly towards regions of high cell density suggesting that the cells are attracted by a chemoattractant secreted by the cells. Therefore, the computational model assumes that cells secrete a chemoattractant to which cells move preferentially. This model produces network-like patterns as shown in Figure 1c. Two important predictions are made based on this model. First, the model predicts an optimal cell density for the formation of stable vascular networks and second, the size of the meshes in the network depend on the diffusivity and decay rate of the chemoattractant.

The mechanical and chemical hypotheses for vascular network formation have also been combined in one mechanochemical model [43]. This continuum model hypothesizes that network formation consists of two stages. First, cells move upwards chemical gradients. Second, at higher local cell density, the cells do not sense the gradient, but the high cell density signals them to start remodeling the matrix. This then attracts cells to the high density regions. The mechanochemical model showed that the assumptions indeed lead to network formation and that chemotaxis 
drives the formation of networks while mechanical interaction stabilize the formed network. In this model matrix elasticity does not affect the properties of the networks. The mechanochemical model is not able to reproduce all observations from both chemical and mechanical angiogenesis models; a more detailed description of the matrix mechanics is required that also influences early cell migration.

Clearly, multiple hypotheses can be used to explain the experimentally observed network formation. Moreover, model observations and predictions for both the mechanical and the chemotaxis model could be reproduced in vitro $[31,36]$. The mechanical models show that matrix thickness and stiffness may be determining factors in network formation, as has been show experimentally [46]. The chemical models reproduce the VEGF dependence that has be observed in vitro [36] as well as a characteristic length of the networks that depends on the diffusivity of the chemoattractant [1]. Both models only produce one similar prediction; there is an optimal cell density for network formation, below this density cells disconnect and above this density cells aggregate [44]. Therefore, it remains unclear whether the two mechanisms are involved in angiogenesis in different environments, or that the two mechanism act consecutive or simultaneously during angiogenesis.

Cell-based models The models discussed so far use a continuum description for both cells and mechanical or chemical fields, meaning that cells and fields are described as densities. This kind of description is appropriate for mechanical and chemical fields; for example, the concentration of a specific chemical can be measured at a specific position and can have any value. However, generalization of cells into cell densities ignores cell behavior, cell properties and cell-cell interactions, which are often key to morphogenic processes such as angiogenesis. Therefore, cells should be the basis of an angiogenesis model. Cell-based models incorporate detailed cell-cell interactions as well as cell properties such as cell shape and size, which can also be measured experimentally for quantification of the parameters and the predictions of the models [27]. Dynamic cell properties and behavior can be added by extending each cell with regulation networks, such as signaling or genetic pathways. Altogether, cell-based models are a solid basis for computational angiogenesis models that can be used to explain tissue effects at the cell level [25].

Different hypotheses have been implemented and compared using cell-based models. One of these models is a hybrid cell-based model, using the Cellular Potts Model (see also Section 3.1), which is based on the assumption that cells chemotact toward a chemoattractant that they themselves secrete [28, 26, 24, 29]. This assumption is similar to the assumption used for the continuum chemotaxis model [36]. In this cell-based model the cells' shape, size and membrane surface are described explicitly, and chemicals are described as continuous fields. One of the main advantages of this cell-based model is the more realistic chemotactic response of cells. This cell based model can be used to simulate network formation solely by defining cell behavior and properties. When only autocrine chemotaxis is implemented, network formation only occurs for narrow parameter ranges: strongly adhering cells or steep chemical gradients [26, 28]. Therefore, Merks et al. proposed two hypotheses for which network formation occurred for a much wider range of parameters: contact inhibition [29] and cell elongation [24]. 
The contact inhibition hypothesis proposes that cells only respond to the autocrine chemoattractants where the cell membrane is not in contact with other cells. This exclusive sensing is thought to be mediated through the dual function of VEcadherin; it acts as a homophilic trans-membrane cell-adhesion molecule and it plays an inhibitor role in the VEGF signaling pathway [9] which increases cell motility. Contact-inhibition reduces the motility locally reduces the cell motility. Therefore, cells within the cluster do not respond to the chemoattractant that all cells secrete. This process appears to contribute to both network formation (Figure 1d) and sprouting angiogenesis. The reasons for this are best understood in the context of sprouting angiogenesis in will therefore be discussed in Section 2.2.

The cell elongation hypothesis is based on the biological observation that cells elongate during network formation. In this model, the combination of elongated cells with autocrine chemotaxis results in network formation [24]. The final network, which can be observed in Figure 1e, is similar to in vitro networks. When cell elongation is omitted, the cells aggregate instead of forming network, indicating that cell elongation drives network formation in this model. The evolution of network properties over time, such as the number of nodes and meshes, correspond with data from in vitro experiments with HUVECs on Matrigel. This suggests that cell elongation may play an important rule during network formation. In this model network formation occurs at two time-scales. First, cell elongation induces a persistent movement along the long axis of the cell. This causes the formation of thin branches of connected cells. Second, the network coarsens by fusion of branches and mesh collapse. This is driven by the chemotaxis that enables slow migration of cells along their short axis.

An alternative hypothesis was proposed by Szabó et al. [42, 41, 40]. Their experiments suggested that neither mechanical interactions nor chemotaxis are required for network formation [42] and that cells move preferential towards elongated cells. From these observation they propose that network formation is driven by the preferential attraction to elongated structures. This hypothesis has been used as a basis for both a particle based model [42] and a cell-based model [40, 41]. In the particle based model cells are represented by point particles that diffuse and adhere to their neighbors. While this model lacks some key cell properties, including cell shape, it suffices as a proof-of-concept model for preferential attraction to elongated structures. The models are used both to investigate network formation from dispersed cells [41] and sprouting from a blob of cells [40]. This model suggests that cells can indeed form network only due to cell-cell interactions, as is shown in Figure 1f. Sprouts formed in these networks only become stable when they connect to other sprouts, suggesting that anastomosis stabilizes the formed network.

Because they all produce similar morphological patterns, none of the modeled hypotheses can be ruled out as a driving force for network formation. Cell-based models $[28,26,24,29]$ suggest that autocrine chemotaxis, combined with cell properties such as contact inhibition of cell elongation, may drive angiogenesis. Other cell-based angiogenesis model $[42,41,40]$ have suggested that autocrine chemotaxis may not be necessary at all. Moreover, mechanical interactions between cells and the matrix have not yet been modeled with a cell-based model. Adding this 
mechanical interaction to cell-based angiogenesis models will help to gain a true understanding of the mechanisms involved in angiogenesis

\subsection{Sprouting}

Sprouting angiogenesis is the formation of new vessels by creating a sprout in the wall of the existing vessel. This form of angiogenesis is often observed in the vicinity of hypoxic tissue that secretes angiogenic factors, e.g., a growing tumor, which activate and attract endothelial cells from the existing vessels [15].

By stimulating the formation of a new vasculature, a tumor is able to grow and proliferate. The mechanisms underlying the dynamics of sprouting angiogenesis are still poorly understood. What mechanisms guide the growing sprout? How do biochemical and biomechanical interactions of the ECM with cells effect sprouting? Is proliferation required and where is proliferation located in the sprout? How are tip cells selected in the vessel and what causes sprouts to branch? Computational models have contributed to a better understanding of these issues.

In corneal angiogenesis sprouting is restricted in absence of proliferation; sprouts will not reach a tumor when cells are not able to divide [38]. A continuum model [2] describes the change in cell density over time due to cell migration driven by cell diffusion, chemotaxis and haptotaxis. The initial configuration of the simulation consists of a blood vessel at one side and a tumor at the other side of the simulation domain. This tumor secretes a chemoattractant, resulting in a gradient of chemoattractant that attracts cells towards the tumor. Haptotaxis is induced by fibronectin that the cells secrete themselves. The highest levels of fibronectin are present where the cell density is maximum. Therefore, haptotaxis and chemotaxis work in opposite directions. The continuum model suggests that, in absence of proliferation, the sprouting is restricted. The authors propose that this is caused because haptotaxis outweighs chemotaxis and increasing the number of cells would increase the chemotactic response.

A problem with this model is that it describes cells as a density field, hence it cannot describe how the sprout breaks up due to lack of proliferation. Therefore, a discrete modeling approach has been introduced to study cell proliferation in the sprout [16]. As illustrated in Figure 2a, the model mimics a cornea with a lesion in the center from which VEGF is secreted. A sprout grows from the periphery and consist of multiple cell types; one leading tip and multiple following stalk cells. The tip cell migrates towards the center induced by the VEGF gradient. Tip cell migration is limited by the elasticity of the tip cell and the strength of the adhesion between stalk cells. Adding proliferation enables unlimited sprout extension. This model suggests that basic cell properties can explain the need for proliferation in sprouting.

The model by Szabó et al. [40] describes cell shape, cell membrane and cell migration in much more detail. The model does not consider chemotaxis or cell-matrix interactions. The cell properties and behavior that are specific for this model are 


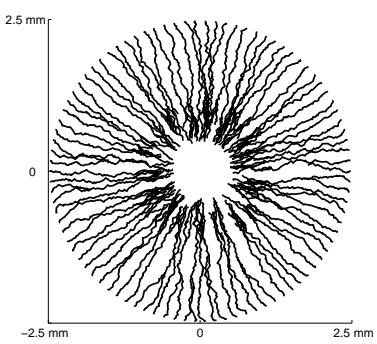

(a)

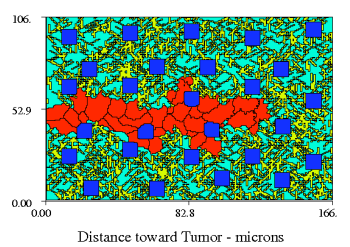

(d)

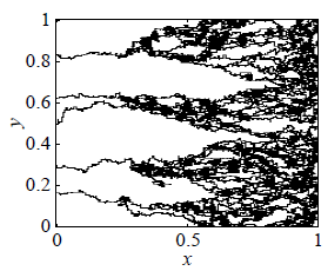

(b)

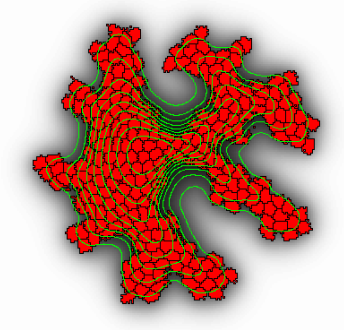

(e)

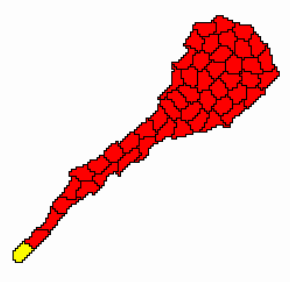

(c)

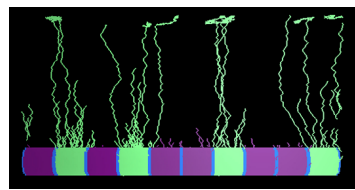

(f)

Fig. 2: Overview of the computational models of angiogenic sprouting. (a) shows corneal angiogenesis as modeled in the discrete model based on tip cell elasticity and stalk cell adhesion [16]. (b) shows the networks formed with the discrete model with chemotaxis and fibrinectin induced haptotaxis [3]. (c) shows sprouting induced by preferential attraction to elongated structures in a cell-based model [40]. (d) shows the outcome of the cell-based model of sprouting angiogenesis in a heterogeneous ECM [4]. (e) illustrates how contact inhibition induces sprouting in the cell-based chemotaxis model [29]. (f) shows how tip cells form sprouts in the agent-based tip cell selection and sprouting model [6]. All images were reproduced with the publishers permission.

preferential attraction to elongated structures, cell polarity and self-propulsion (i.e. persistence of motion). The model also differentiates between tip and stalk cells. The tip cell is polarized, causing directed movement in the direction of the polarization vector. The results shown in Figure $2 \mathrm{c}$ suggest that both preferential attraction and self-propulsion are necessary to reproduce realistic sprouting behavior. Cell polarization may be regulated by cell-cell contacts and VE-cadherin may be a key player for this. Moreover, the model suggest that differential behavior at the tip of the sprout may drive sprout formation. Therefore, this model suggests that proliferation may not be required, as long as the supply of cells from the main vessel is sufficient.

Cell-matrix interactions The previous two cell-based sprouting models have only considered cell properties and cell behavior, ignoring all ECM and stromal tissue. Anderson and coworkers [3] created a particle based, hybrid model describing sprouting angiogenesis. In this model cells are represented as point particles on a grid while the chemotactic and haptotactic fields are still described as continuum equations. This model was used to investigate how the balance of haptotaxis and chemotaxis influences branching and anastomosis. As shown in Figure $2 b$ branching 
and anastomosis occur in the model, but these behaviors only occur when cells are able to move perpendicular to the chemotactic field, which is enabled by haptotaxis. When the haptotactic forces are strong enough branches can split and reconnect in order to form a functional vasculature.

Anderson et al.'s model [3] suggests that haptotaxis is key to branching, but it did not show how cells interact with their heterogeneous environment. A more recent, cell-based model, represents the ECM as a static, heterogeneous configuration of matrix fiber bundles, interstitial fluid and immobile tissue-specific cells [4]. The endothelial cells in the model are motile and adhere stronger to matrix fibers than to the surrounding matrix. Immobile cells act as obstacles that hinder the migration of endothelial cells. The tip cell is influenced by a chemoattractant field and it degrades ECM components. Degradation of the extracellular matrix during sprouting enables cells to migrate and branch off the main sprout as shown in Figure 2d. The model suggests that a heterogeneous composition of the matrix is necessary for the formation of branches; the inhomogeneities in the matrix enable cells to split from the main branch. Furthermore, the model suggests that the proliferation region determines sprouting dynamics but does not affect the final sprout morphology.

A follow-up model was used to investigate cell-ECM interaction in more detail [5]. In this model all cells respond to the chemoattractant and that the immobile tissue cells are removed, i.e., only fibers cause matrix heterogeneity. The model suggests that sprouting only occurs in a specific range of matrix densities, which corresponds with experimental observations. Moreover, simulation results suggest that low fiber density results in cell elongation. Similar changes were observed when the random fibers were replaced by a specific fiber pattern, for example long fibers cause cells to elongate in the same direction as the fibers. The authors propose that contact guidance, due to cell-matrix interactions, is key to role in vascular sprouting because it enables sprout branching in an inhomogeneous matrix.

Another model suggests branching can occur in the absence of matrix heterogeneity [29]. The model assumes cells are attracted towards an autocrine chemoattractant, using similar rules as in chemotaxis-based network model (Section 2.1). Contact inhibition mediated by VE-cadherin causes cells to be only sensitive to the chemoattractant at positions of the cell membrane adjacent to the ECM. Sprouting occurs in two ways. First, when cells are arranged in an aggregate, only the outer layer of cells sense the chemoattractant. These cells tend to migrate towards the center of the aggregate causing a buckling instability that induces sprouting. This effect enables cells, even those with a low motility, to move against the chemotactic gradient. Second, another mechanism may explain sprouting for highly motile cells. To move away from the mother vessel and form a sprout, cells must migrate against a steep gradient of self-secreted chemoattractant. Once a small sprout is created by a motile cell, the gradient around this outgrowth is less steep than the rest of the gradient, so cells within the sprout have higher motility than elsewhere, causing an instability.

Although all previous models simplified angiogenesis by assuming endothelial cells all have identical properties, in fact differentiation between leading "tip" cells and following "stalk" cells is key to sprouting angiogenesis. Bentley et al. [6] inves- 
tigate the molecular and biophysical mechanisms driving tip and stalk cell differentiation using an agent-based, computational model [6]. The model represents a row of cylindrical endothelial tip and stalk cells made up of multiple agents. Only the tip cells can extend filopodia outwards, representing an new sprout as shown in Figure $2 \mathrm{f}$. The model is used to study the interaction between Dll4-Notch1-signaling with VEGF-induces tip cell activation [14]. The robustness of tip cell selection is investigated by applying a VEGF gradient perpendicular to the vessel [7] ; each cell senses the same level of VEGF, which combined with a Dll4-Notch1-based lateral inhibition mechanism produces a pattern of alternating tip and stalk cells. The tip cells grow long filopodia that may meet up to form a connected vessel; anastomosis. When the common surface area of the connecting tip cells has increased sufficiently, one of the two cells becomes a stalk cells and the vessel stabilizes. Thus, the model suggests that the common surface area is a determining factor for tip cells selection; if the common surface area is too small lateral inhibition does not work. A second application of the model involved cellular competition for the tip cell position. Time-lapse microscopy has shown how stalk cells migrate along the sprout, take up the role of tip cell, and inhibit the original tip cell become a stalk cell [17]. Cell variants with higher levels of VEGFR2-expression have a competitive advantage over the wild-types: they end up more often at the tip of the sprout, but only if Notch1 can inhibit Dll4 expression. The advantage of the variants diminishes when all cells have low levels of Notch1. These observations suggest that Notch limits the levels of VEGFR2 in wild-type cells. Bentley test this hypothesis in her agent-based angiogenesis model by applying a VEGF gradient along the sprout and by allowing cells to switch places. Switching is regulated the level of VEGFR2 and Notch expression; VEGFR2 promotes switches towards VEGF while Notch1 inhibits the same switches. With these assumptions the experimental observation could be reproduced, suggesting that this mechanism may explain tip cell shuffling.

\subsection{The future of angiogenesis modeling}

The models discussed sofar, all isolated specific aspects of angiogenesis to predict the outcome of proposed in vitro experiments. To study angiogenesis in vivo, we must incorporate the interaction with the rest of the body in a multi-scale model. Angiogenesis is induced by hypoxic tissue which, for example, can be a tumor or an active muscle. The change in oxygen and nutrient supply due to the new vasculature changes the signals coming from the tissue, resulting in a dynamic feedback loop between angiogenesis and the needs of the tissue. Also blood flow may be key to this feedback. Disfunctional vessels are not able to support blood flow and do not contribute to the perfusion of the tissue. Endothelial cell change their behavior due to the shear stresses induced by blood flow [10]. The inclusion of these processes in a multi-scale angiogenesis model would be a great tool to study whether pathological processes either involve excessive or insufficient blood vessel growth. Such multi-scale models can not only be used to formalize and validate hypotheses, they 
can also be used to predict the effects of pro- or anti-angiogenic therapies on the vasculature and the other tissues involved.

In order to build these multi-scale models, researchers often extend existing models. For example, the particle-based sprouting model by Anderson et al. [3] has been extended with blood flow [23]. This model suggested that most vessels are not perfused due to the lack of anastomosis, and thus drugs can not reach the target. More complex approaches have been used to combine more detailed angiogenesis models with blood flow and the kinetics of oxygen and VEGF [33, 34, 32]. This model has show to produce vascularization similar to experimental observation in a heterogeneous extracellular matrix [34] and in the skeletal muscle [21].

In the previous models the surroundings of the vasculature are static and are not being changed by the growing vessels and the increasing supply of oxygen and nutrients. This means that a part of the feedback is missing, for example a tumor can grow when the blood supply increases and a larger tumor needs a bigger supply of blood. Shirinifard et al. [37] combined cell-based Cellular Potts models (see Section 3.1) of blood vessel formation and tumor growth to investigate how tumor growth and vascular remodeling interact. This high level of detail gives insight in how specific cell properties influence tumor growth and angiogenesis.

Cell-based modeling would be a suitable approach to create predictive multiscale models. Cell behavior in such a model must be linked to biological or physical cell properties. The extracellular matrix as well as blood flow could be added to the model. Then, the cell properties could be linked to matrix interactions and local levels of oxygen, nutrients and other chemicals. A cell-based model could simulate emergent angiogenesis and blood vessel remodeling and could be used to predict the effects of therapeutic agents.

\section{Cell-based model of in vitro sprouting}

The previous section discussed how multi-level computational models aim to fuse models to incorporate different aspects of angiogenesis, such as cell behavior, matrix interactions and blood flow. Processes like chemotaxis and haptotaxis can be described with continuum models, while we argued that the representation of cells requires a cell-based approach. Cell-based models explicitly model cell structures (e.g. cell membranes) and cell behaviors (e.g. cell-cell adhesion and pseudopod extensions). These models are intuitive and relate well with biological observations. To illustrate the approach, we created a computational cell-based model of an in vitro model of capillary-like tube formation, introduced by Koolwijk et al. [19].

A schematic representation of a cross-section of the experimental model is given in Figure 3. A monolayer of isolated human microvascular endothelial cells (hMVEC) is seeded on a three-dimensional fibrin matrix. The composition of this matrix, consisting of only fibrin (Figure 7), is completely controlled and reproducible. The endothelial cells grow into the matrix and form capillary-like tubular structures upon stimulation with an angiogenic factor, VEGF and/or bFGF (basic 


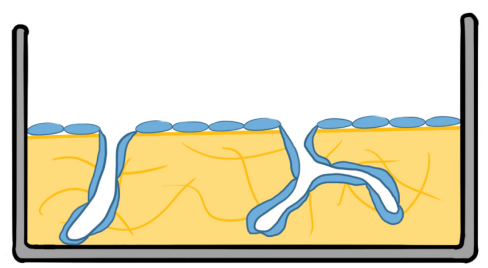

Fig. 3: A schematic cross-section of the experimental model by Koolwijk et al. [19]. Endothelial cells are seeded on a three-dimensional fibrin matrix and form capillary-like tubular structures upon stimulation.

fibroblast growth factor), in combination with the inflammatory mediator TNF $\alpha$ (tumor-necrosis factor alpha). TNF $\alpha$ is suggested to induce receptor-bound u-PA (urokinase-type plasminogen activator) activity to enable the cells to degrade the fibrin matrix [45]. Although VEGF and bFGF are both growth factors, TNF $\alpha$ completely inhibited the growth factor-induced proliferation in this experimental model.

The computational model of sprouting is designed to be easy to relate to the experimental observations. The model can be used to explain and predict angiogenic patterning on tissue level, based on quantitative descriptions of cell behavior. The most important cell behaviors, such as cell shape, cell adhesion and haptotaxis are therefore included, and their effects and relative importance in sprouting can be examined. Endothelial cells have a wide range of interactions with the extracellular matrix, of which the function as well the effects are often still unclear. Endothelial cells for instance secrete proteolytic enzymes to degrade the matrix. The proteolytic activity is regulated by a complex system and the computational model can help to gain insight in the relative importance of the components in this system, in the regulation of the system and in the effect of this regulation on sprouting.

\subsection{Computational sprouting model}

To mimic the experimental set-up of Koolwijk et al., the computational model starts with a monolayer of cells, of which one is a tip cell and the rest are stalk cells, on a fibrin matrix and a basement membrane (BM) in between. The Cellular Potts model $[12,13]$ describes the shape, behavior and movement of the cells. The tip cell will secrete proteolytic enzymes, MMPs (matrix metalloproteinases) and u-PA, to degrade the BM and fibrin respectively. The endothelial cells are attracted to higher concentrations of fibrin, which is also modeled as a concentration field, and migrate into the created space to form a sprout. On top, a local chemorepulsive field exerts a small pressure on the endothelial cells in the monolayer to keep them flattened and well connected. A schematic overview of the model is given in Figure 4. 


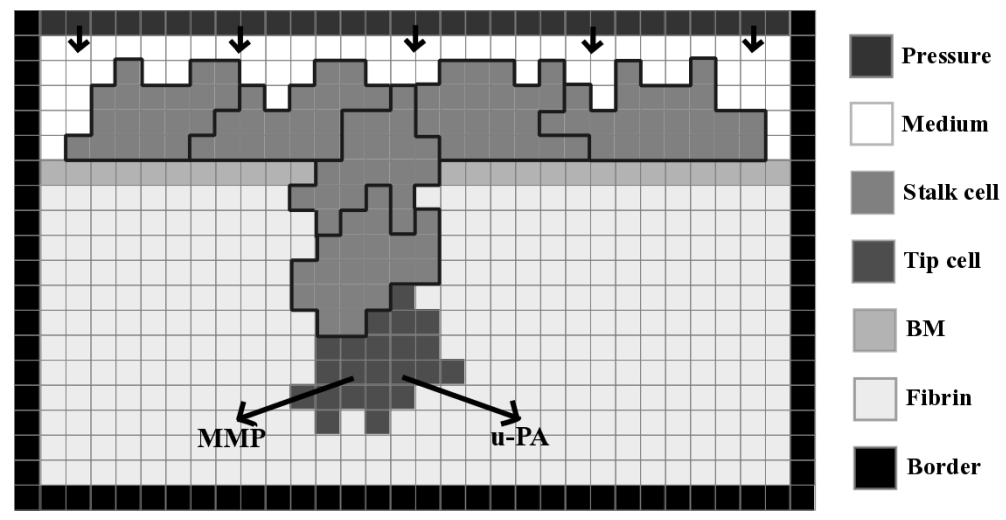

Fig. 4: Schematic representation of the Cellular Potts model. The different colors depict the types defined in the sprouting model. The tip cell secretes MMP and u-PA (large arrows) to degrade the extracellular matrix and the small arrows on top represent a the small chemorepulsive pressure on the cells.

The Cellular Potts model represents cells, BM and fibrin as patches of grid sites (Figure 4), which are differentiated by types $(\tau)$ with specific properties and behaviors. Cells can migrate by the addition and removal of grid sites at their cell membranes, which can be seen as extensions or retractions of pseudopodia. A grid site (x) is added or removed by copying the contents of a neighboring grid site ( $\mathbf{x}^{\prime}$ ) (Figure 5). Whether such a copy is allowed depends on an energy function, which summarizes the balance of forces resulting from cell behavior: $\mathrm{E}=$ $\mathrm{E}_{\text {contact }}+\mathrm{E}_{\text {shape }}+\mathrm{E}_{\text {connectivity. }}$. The change in this local energy $(\Delta \mathrm{E})$ for a certain copy is a measure for its favorability. Energy decreasing copies will always be accepted; while a copy that increases energy will be accepted according to a Boltzmann probability function: $\mathrm{P}_{\text {accept }}(\Delta \mathrm{E})=e^{\frac{-\Delta E}{\mu}}$. The ability to accept copies that cost energy resembles active cell motility, with the parameter $\mu$ a cell motility parameter.

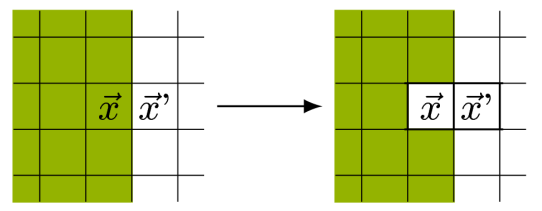

Fig. 5: Graphical representation of a copy attempt. A random grid site $(\mathbf{x})$ is chosen to copy the state of a neighboring grid site $\left(\mathbf{x}^{\prime}\right)$ to simulate pseudopod extensions and retractions.

Adhesion or repulsion by cells is modeled by contact energy between types $\left(J_{\tau 1, \tau 2}\right)$ : lower energies resemble stronger adhesions between types. The model is surrounded by a border (Figure 4) which has high contact energy with cells to prevent them from sticking to the edges. The overall contact energy sums the contact energies between cells and between cells and their surrounding: 


$$
\mathrm{E}_{\mathrm{contact}}=\sum_{\left(\mathbf{x}, \mathbf{x}^{\prime}\right)} J_{\tau(\mathbf{x}), \tau\left(\mathbf{x}^{\prime}\right)}
$$

with $\left(\mathbf{x}, \mathbf{x}^{\prime}\right)$ a pair of adjacent grid sites at the cell membrane. Migrating cells have a typical shape, depending on cell size (area) and membrane surface (perimeter). Therefore, the energy increases with the deviation of a target value. $\lambda_{\text {area }}$ and

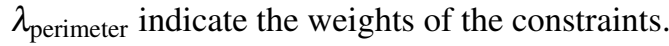

$$
\mathrm{E}_{\text {shape }}=\sum_{c \in \text { cells }} \lambda_{\text {area }}\left(\tau_{c}\right)\left(A(c)-A_{\text {target }}\left(\tau_{c}\right)\right)^{2}+\lambda_{\text {perimeter }}\left(\tau_{c}\right)\left(P(c)-P_{\text {target }}\left(\tau_{c}\right)\right)^{2}
$$

Biological cells do not break up, thus all pixels of one cell must be connected. Therefore, a large penalty energy ( $\mathrm{E}_{\text {connectivity }}$; see Table 1$)$ is added to the energy function when a tip or stalk cell has lost its connectivity [39].

The tip and stalk cell are surrounded by extracellular matrix, that is fibrin or BM. Unlike the migrating cells, fibrin and BM are immobile types. Endothelial cells can preferentially migrate towards higher concentrations of adhesion sites in the extracellular matrix, this process is called haptotaxis. In order to model this, fibrin and $\mathrm{BM}$ are also modeled as static homogeneous concentration fields that attract tip and stalk cells. The tip cell secretes u-PA and MMP to locally degrade the concentration fields. The probability that the types 'fibrin' and 'BM' are degraded depends on their local field concentrations by a Hill equation: $\mathrm{P}_{\text {degradation of } \mathrm{X}}=\frac{[X]^{n}}{[k]^{n}+[X]^{n}}$. The concentrations of the proteolytic enzymes are described by the following partial differential equation: $\frac{\delta c}{\delta t}=D \nabla^{2} c+k c+s_{\tau}$, where $D$ refers to the diffusion constant, $k$ to the decay constant and $s$ to the secretion of the enzyme $c$ by type $\tau$. The local degradation of the haptotactic fields by the secreted proteolytic enzymes results in concentration gradients, a cell will preferentially extend up the gradient [35]. The effect of haptotaxis is calculated for every copy attempt. Chemotaxis and haptotaxis are implemented as:

$$
\Delta \mathrm{E}=\mathrm{E}_{\text {new }}-\mathrm{E}_{\text {old }}-\Delta \mathrm{E}_{\text {haptotaxis }}, \text { where } \Delta \mathrm{E}_{\text {haptotaxis }}=\lambda_{\text {haptotaxis }}\left(c\left(\mathbf{x}^{\prime}\right)-c(\mathbf{x})\right),
$$

with $c$ the concentration of the attracting component and $\lambda_{\text {haptotaxis }}$ describes the weight of the constraint.

\subsection{Integration of the experimental and computational model}

In section 3.1, we showed how a conceptual model of tube formation translates to a computational model. Basic cell behaviors and properties are included in the computational and the default parameters can be found in Table 1. First, the model is used to test a hypothesis concerning the relation of proteolytic enzyme secretion and sprout morphology. Subsequently, the model will be extended, mainly focusing on matrix interactions, to differentiate between forces that drive migration. With 
Table 1: Default parameters for the sprouting model.

\begin{tabular}{ll}
\hline Property & Value \\
\hline Contact energy & $J_{\text {stalk,medium }}=1, J_{\text {stalk,tip }}=1, J_{\text {tip, BM }}=100$ \\
& $J_{\text {stalk,border }}=10^{7}, J_{\text {stalk,pressure }}=10^{7}, J_{\text {tip, border }}=10^{7}, J_{\text {tip,pressure }}=10^{7}$ \\
Area & $A_{\text {target }}($ tip,stalk $)=50, \lambda($ tip,stalk $)=70$ \\
Perimeter & $P_{\text {target }}($ tip,stalk $)=30, \lambda($ tip $)=25, \lambda($ stalk $)=5$ \\
Haptotaxis & $\lambda($ tip,stalk $)=1000$ \\
Chemotactic pressure & $\lambda($ tip,stalk $)=-0.1$ \\
Pressure field & $D=10^{-2}, k=0.01, s=1000$ \\
MMP and u-PA field & $D=10^{-6}, k=0.01, s=500$ \\
Fibrin degradation & type: $n=4, k=1.9$, initial concentration $=2$ \\
& field: $-10^{-7} \cdot[\mathrm{u}-\mathrm{PA}][$ fibrin $]$ \\
BM degradation & type: $n=3, k=1$, initial concentration $=2$ \\
& field: $-5 * 10^{-8} \cdot[\mathrm{MMP}][\mathrm{BM}]$ \\
\hline
\end{tabular}

these examples, we emphasize how computational and experimental biologists can benefit from each other's models in their quest to understand angiogenesis.

\subsubsection{Matrix degradation and sprout morphology}

To induce sprouting, endothelial cells are stimulated with an angiogenic factor, VEGF and/or bFGF, in combination with the inflammatory mediator TNF $\alpha[19,8]$. Both the angiogenic factors as well as $\mathrm{TNF} \alpha$ induce proteolytic enzyme activity. The growth factors are suggested to stimulate secretion of a soluble form of plasminogen activators, t-PA (tissue plasminogen activator), which becomes active upon contact with fibrin and degrades fibrin in a diffuse manner. TNF $\alpha$ is suggested to induce u-PA (urokinase-type plasminogen activator) production and thereby stimulate receptor-bound u-PA activity [19]. u-PA is inactive until it is bound to its membranebound receptor, which localizes proteolytic activity to the membranes of the receptor expressing cells. Besides u-PA, TNF $\alpha$ can also induce the plasminogen activator inhibitor, PAI-1, which inhibits u-PA and t-PA. Proteolytic activity during sprouting is closely regulated by endothelial cells and results from a balance between the proteolytic enzymes and their inhibitors. Endothelial cells secrete MMPs (matrix metalloproteinases) to degrade the basement membrane. The production and activity of u-PA and MMPs is interlinked; u-PA activity is suggested to induce MMP activity indirectly and at least one membrane-bound MMP (MT1-MMP) is known to be capable of fibrin degradation as well [47].

Proteolytic activity can be manipulated by stimulation the cells with angiogenic factors or inflammatory factors [8]. To induce sprout formation, low amounts of $\mathrm{TNF} \alpha$ are added to the monolayer of endothelial cells in combination with a growth factor. Stimulation with angiogenic factors alone induces a uniform degradation of fibrin and the monolayer of endothelial cells does not sprout but as a result lowers as a whole. This uniform degradation of fibrin can result from a combination of diffuse proteolysis induced by the angiogenic factors and the absence of inflamma- 
tory factor induced inhibitors of proteolysis. Excessive plasminogen activation, in endothelial cells seeded in suspension into a three-dimensional fibrin matrix, results in the formation of round cyst-like structures [30]. Cyst-like structures are also observed in the endothelial monolayer model [19] after stimulation with a higher dose of TNF $\alpha$ in combination with angiogenic growth factors by Koolwijk et al. (data not shown).

We aim to find the conditions that is suffice to explain the experimental observations on sprout morphology. This hypothesis will then be tested with the computational model. We hypothesize that the intensity of proteolytic enzyme secretion (u-PA and MMP) as well as the distribution of secretion over different cell types is responsible for the observed phenotypes in angiogenesis. After low stimulation with $\mathrm{TNF} \alpha$, tip cells might already secrete proteolytic enzymes at maximal rate and are therefore insensitive to stimulation. In contrast, stalk cells normally do not secrete many proteolytic enzymes and are therefore more sensitive for stimulation than tip cells. Indeed, the tip cell was seen to have the most u-PA receptors during sprouting, which facilitate proteolysis to degrade fibrin [20]. When both tip and stalk cells secrete high levels of proteolytic enzymes, the fibrin will be degraded uniformly.

To test this hypothesis in the computational model, we assumed that both tip and stalk cells can secrete u-PA and MMP. As discussed above, proteolysis is a complex system that involves membrane-bound and soluble proteolytic enzymes as well as inhibitors. For simplicity, only membrane associated proteolytic activity and no inhibitors are considered in this first attempt. Since inhibitors are not modeled explicitly, proteolytic activity and secretion of proteolytic enzymes are directly coupled. The presence of inhibitor is modeled by lower secretion rates of the proteolytic enzyme. To model proteolytic activity at the membrane, the diffusion constants for the proteolytic enzymes are set to a very low value (Table 1). Secretion of the proteolytic enzymes could also be thought of as expression of the receptors that facilitate the activity of the enzymes at the membrane. Soluble proteolytic enzymes are not modeled, although high secretion of proteolytic enzymes does result in proteolytic activity at a larger distance. MMPs are assumed to exclusively degrade the basement membrane, while u-PA degrades the fibrin matrix. Mitosis of stalk cells is also included in the model to supply the monolayer with new cells, simply by dividing a cell over its short axis when it has increased by an arbitrary factor of 1.28. The secretion of the proteolytic enzymes by the tip cell is kept constant at a maximal rate.

We study how u-PA and MMP secretion by stalk cells affects sprout morphology. Figure 6 gives an overview of the simulation results as a function of the secretion rates of u-PA and MMP. Because the simulations are stochastic, variation is seen between simulations with the same parameter settings; representative simulations were selected for the morphospace in Figure 6. Sprout morphology can be grouped in four categories: sprouts, solid round cysts, hollow cysts and monolayers. Sprouts have a cord-like orientation of cells, while cysts are more round and multi-cellular. A high secretion of both u-PA and MMP (Figure 6D) by stalk cells results in lowering of the monolayer. Sprouts are formed for low secretion of u-PA (Figure 6A), while solid cyst-like structures are formed for medium levels of u-PA secretion (Figure 6B) 


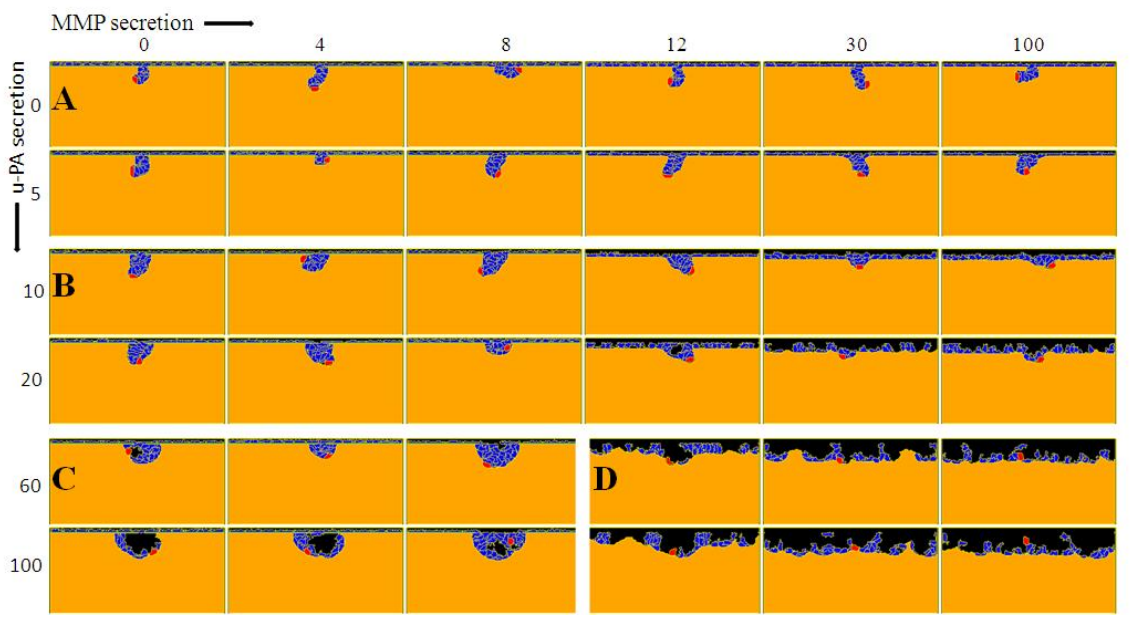

Fig. 6: Morphospace of proteolytic enzyme secretion by stalk cells. Tip and stalk cells secrete u-PA and MMP to degrade fibrin and the basement membrane respectively. The secretion of u-PA and MMP by stalk cells is expressed in percentage of the maximal secretion rate as secreted by the tip cell. This results in different sprout morphologies: sprouts (A), solid cyst-like structures (B), hollow cyst-like structures (C) and monolayers (D).

for all MMP secretion levels by stalk cells. High secretion of u-PA and low secretion of MMP (Figure 6C) results in hollow-cyst like structures. Occasionally (6 out of 128 simulations), no sprouting occurs for low levels of MMP secretion by stalk cells because stalk cells position themselves between the tip cell and the BM and thereby prevent degradation of the basement membrane. An interesting transition is seen between a hollow cyst-like structure and monolayer lowering for a secretion of MMP between $8 \%$ and $12 \%$. The hollow cyst-like structures can be formed since the BM remains intact for attachment of endothelial cells before this transition. These structures are likely to collapse if gravity was included in the model. Experimentally, tubular structures can also disappear due to excessive fibrinolysis [8].

u-PA can activate MMP, thus the activity of both enzymes are likely to increase simultaneously. The sprout morphologies that are seen along diagonals of the morphospace in Figure 6 are therefore biologically most probable. Along the diagonal, we see sprout formation for low secretion of both enzymes by stalk cells, cyst formation for medium secretion and monolayer lowering for high secretion. This is consistent with the experimental results by Collen et al. [8] and our own as discussed above. The intensity and the distribution of proteolytic enzyme secretion over different cell types (tip and stalk cells) thus seems to be a sufficient explanation for the tissue behavior observed in the laboratory.

The computational model is oversimplified, because it only describes proteolytic activity at the membrane and no diffuse matrix degradation or inhibitors. The notion that $\mathrm{TNF} \alpha$ induces secretion of proteolytic enzymes as well as their inhibitors implicates a more complex regulation of matrix degradation during sprouting. Low- 
ering of the endothelial monolayer, when exclusively stimulated with angiogenic factors, is likely to depend on soluble rather than membrane-bound proteolytic enzymes. Inhibitors and soluble proteolytic enzymes should be included to understand the basic principles of angiogenesis.
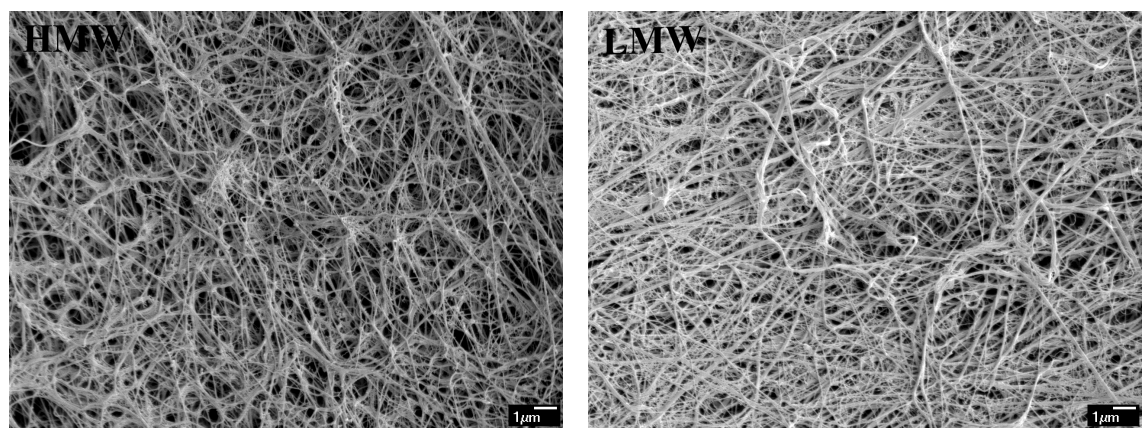

Fig. 7: Fibrin matrices. Scanning electron microscopic analysis of fibrin networks. High molecular weight (HMW) fibrinogen is thicker and forms more open network structures (left Figure) than low molecular weight (LMW) fibrinogen (right Figure). Bars represent $1 \mu \mathrm{m}$.

Beside proteolytic degradation of the extracellular matrix, it is likely that the structure of the matrix influences tube formation. Matrix structure for example effects the sensitivity of fibrin matrices to proteolytic degradation [8]. The composition of fibrin matrices can be controlled experimentally [18]. High molecular weight (HMW) and low molecular weight (LMW) fibrinogen can be isolated from unfractionated fibrin and used to create different densities of the matrix. A matrix composed of only LMW fibrinogen has thinner fibers and has a denser structure than a matrix made from only HMW fibrinogen (Figure 7). Small differences in fibrin matrix lead to different gene expression patterns in endothelial cells [47] and influence tube formation [18]. Tube formation was more extensive in HMW than in LMW matrices. We plan to use the computational model to examine how these differences in matrix structure influence sprouting.

\subsection{Modeling perspectives}

Koolwijk et al. developed a simple in vitro model to examine tube formation in angiogenesis [19]. To gain knowledge in the mechanism and key players involved, we created a computational model that resembles the experimental in vitro model. The computational model is used to validate conceptual models for basic mechanisms of sprouting and to predict how alterations in cell behavior will affect sprouting. The model explains experimental observations concerning the effects of proteolytic enzyme activity on sprout morphology by supporting the hypothesis that not only the 
level, but also the distribution of proteolytic enzyme secretion over tip and stalk cells is responsible for different sprout morphologies. Several experimental observations and techniques can help to validate conclusions drawn from the model. Contradictions between experimental data and the computational predictions point out gaps in our understanding. A clear focus point for improvement is the structure of the extracellular matrix and the interactions of cells with the matrix.

We currently model the extracellular matrix in more detail. Endothelial cells strongly interact with the matrix, mechanically as well as chemically. Cells can adhere to the fibers in the matrix and rearrange the fibers. By modeling the actual fibers and their alignment, physical obstruction of the matrix, haptotaxis and directional guidance of migration can be considered. Endothelial cells can also degrade the matrix with a sensitive and complicated system of proteolytic enzymes. Proteolysis of the matrix locally releases chemotactic components that stimulate sprouting. Each of these mechanical, haptotactical and chemotactical forces resulting from the surrounding tissue influence angiogenesis and are therefore important to understand.

Besides modeling the extracellular matrix more intensively, the interactions within the cell should be focused on. Endothelial cells adhere to the extracellular matrix with integrins, which are also linked to the cytoskeleton of the cell. By modeling cytoskeleton remodeling and integrin-mediated binding at a molecular level, we can study mechanical matrix interaction. Additionally, tip cell selection is an interesting molecular interaction within and between cells to include in the model. Tip cell selection depends on Delta-Notch signaling [14] and is required for experimentally observed branching of tubes.

In conclusion, we aim to create a multi-level model of angiogenesis that includes the molecular, cellular and tissue level. Each level should be modeled simple and intuitively and the interaction between the levels must be taken in close consideration. To understand which components should be modeled and how they interact, a continuous feedback between experimental and computational modelers is needed.

\section{Conclusion}

In this chapter, we have reviewed how computational models shed new light on questions involving network formation and sprouting. Firstly, we discussed how computational models are used to test alternative hypotheses on the mechanisms that drive network formation. Cellular and environmental factors in these models are studied to predict their effects on angiogenesis. A comparison of experimental results and these computational predictions can show which mechanisms are most likely the driving forces of network formation. Secondly, we focused on computational models that provide new insights in the mechanisms of sprouting. These models shed light on questions about the regulation of sprouting, such as the necessity and location of proliferation, dynamics of tip cell selection, and the influence of angiogenic factors and the extracellular matrix. The discussed models use different techniques to model cells: continuum models describe cells as densities, while 
discrete models represent them as particles. The functioning of endothelial cells dependents on thousands of interacting proteins and genes. Cell-based models are discrete models that represent the results of these gene and protein interactions by a set of cell properties (e.g. cell and membrane size) and behaviors (e.g. adhesion and chemotaxis), suggesting that a few cell behaviors sometimes suffice to explain complex collective cell behaviors like angiogenesis [25].

To illustrate this approach in more detail, we created a cell-based model to study angiogenic sprouting. This computational model is based on an in vitro model of sprouting in a fibrin matrix by Koolwijk et al. [19]. The model is used to formalize the mechanisms that are minimally required for sprouting and it predicts the effects on the dynamics of tube formation of varying cell properties, such as matrix degradation. Predictions from the model can lead to new insights and drive experimental research. The observations and results from the experimental research are crucial for the validation and further development of the computational model. A focus point for further study is the interaction of endothelial cells with the extracellular matrix. Various interaction with the matrix strongly influences sprouting, but it is difficult to separately study them experimentally. We plan to model the extracellular matrix itself and its interactions with endothelial cells in more detail and extend our model to a multi-scale model, including molecular, cellular and tissue levels, to gain insight in these interactions. In a close cooperation between experimental and computational biologists, we can reach a thorough understanding of how the interactions between multiple levels of organization lead to counterintuitive effects, which experiments alone would not unveil.

Multi-scale modeling is thought to be the next step in computational modeling. If different scales and their interactions are modeled simultaneously, we can identify the global and local (side) effects of a therapeutic drug. Some multi-scale models of angiogenesis have already been developed [23, 33, 34, 32, 21, 37], as discussed in section 2. So far, many of these models are based on phenomenological rules and the results are direct results of the implemented rules. In order to make the step to explanatory rather than descriptive multi-scale models, a thorough understanding of the mechanisms at the separate levels is needed. A few international projects recognize this need for explanatory multi-scale models, like the Physiome Project and the Virtual Physiological Human Project [21]. We argue that cells and their behavior should still have a central role in these models, since cell behaviors and properties can also be observed and measured experimentally, which allows validation and quantification of the computational model. A constant feedback loop between computational and experimental models is thus needed to reach a functional and multi-level understanding of angiogenesis, a strategy called systems biology.

\section{Acknowledgements}

We thank Indiana University and the Biocomplexity Institute for providing the CC3D modeling environment. This work was cofinanced by the Netherlands Con- 
sortium for Systems Biology (NCSB) which is part of the Netherlands Genomics Initiative / Netherlands organization for Scientific Research and by the Netherlands Institute of Regenerative Medicine. The investigations were (in part) supported by the Division for Earth and Life Sciences (ALW) with financial aid from the Netherlands Organization for Scientific Research (NWO).

\section{References}

1. Ambrosi, D., Gamba, a., Serini, G.: Cell directional persistence and chemotaxis in vascular morphogenesis. Bull. Math. Biol. 66(6), 1851-73 (2004). DOI 10.1016/j.bulm.2004.04.004

2. Anderson, A., Chaplain, M.: A mathematical model for capillary network formation in the absence of endothelial cell proliferation. Appl. Math. Lett. 11(3), 109-114 (1998)

3. Anderson, A., Chaplain, M.: Continuous and discrete mathematical models of tumor-induced angiogenesis. Bull. Math. Biol. 60(5), 857-899 (1998). DOI 10.1006/bulm.1998.0042

4. Bauer, A., Jackson, T., Jiang, Y.: A cell-based model exhibiting branching and anastomosis during tumor-induced angiogenesis. Biophys. J. 92(9), 3105-3121 (2007)

5. Bauer, A., Jackson, T., Jiang, Y.: Topography of extracellular matrix mediates vascular morphogenesis and migration speeds in angiogenesis. PLoS Comput. Biol. 5(7), e1000,445 (2009). DOI 10.1371/journal.pcbi.1000445

6. Bentley, K., Gerhardt, H., Bates, P.: Agent-based simulation of notch-mediated tip cell selection in angiogenic sprout initialisation. J. Theor. Biol. 250(1), 25-36 (2008). DOI 10.1016/j.jtbi.2007.09.015

7. Bentley, K., Mariggi, G., Gerhardt, H., Bates, P.: Tipping the balance: robustness of tip cell selection, migration and fusion in angiogenesis. PLoS Comput. Biol. 5(10) (2009). DOI 10.1371/journal.pcbi.1000549

8. Collen, A., Koolwijk, P., Kroon, M., van Hinsbergh, V.: Influence of fibrin structure on the formation and maintenance of capillary-like tubules by human microvascular endothelial cells. Angiogenesis 2(2), 153-166 (1998)

9. Dejana, E.: Endothelial cell-cell junctions: happy together. Nature Reviews Molecular Cell Biology 5, 261-270 (2004)

10. Fisher, A., Chien, S., Barakat, A.: Endothelial cellular response to altered shear stress. Am. J. Physiol.-Lung. C. 281(3), L529-L533 (2001). URL http://ajplung.physiology.org/content/281/3/L529.short

11. Gamba, A., Ambrosi, D., Coniglio, A., De Candia, A., Di Talia, S., Giraudo, E., Serini, G., Preziosi, L., Bussolino, F.: Percolation, morphogenesis, and Burgers dynamics in blood vessels formation. Phys. Rev. Lett. 90(11), 118,101 (2003)

12. Glazier, J.A., Graner, F.: Simulation of the differential adhesion driven rearrangement of biological cells. Phys. Rev. E: Stat. Phys., Plasmas, Fluids, Relat. Interdiscip. Top. 47(3), 21282154 (1993)

13. Graner, F., Glazier, J.: Simulation of biological cell sorting using a two-dimensional extended Potts model. Phys. Rev. Lett. 69(13), 2013-2016 (1992)

14. Hellström, M., Phng, L.K., Hofmann, J.J., Wallgard, E., Coultas, L., Lindblom, P., Alva, J., Nilsson, A.K., Karlsson, L., Gaiano, N., Yoon, K., Rossant, J., Iruela-Arispe, M.L., Kalén, M., Gerhardt, H., Betsholtz, C.: Dl14 signalling through Notch1 regulates formation of tip cells during angiogenesis. Nature 445(7129), 776-80 (2007). DOI 10.1038/nature05571

15. Hillen, F., Griffioen, A.W.: Tumour vascularization: sprouting angiogenesis and beyond. Cancer Metastasis Rev. 26(3-4), 489-502 (2007). DOI 10.1007/s10555-007-9094-7

16. Jackson, T., Zheng, X.: A Cell-based Model of Endothelial Cell Migration, Proliferation and Maturation During Corneal Angiogenesis. Bull. Math. Biol. (2010). DOI 10.1007/s11538009-9471-1 
17. Jakobsson, L., Franco, C., Bentley, K., Collins, R., Ponsioen, B., Aspalter, I., Rosewell, I., Busse, M., Thurston, G., Medvinsky, A., Schulte-Merker, S., Gerhardt, H.: Endothelial cells dynamically compete for the tip cell position during angiogenic sprouting. Nature cell biology 12(10), 943-953 (2010). DOI 10.1038/ncb2103

18. Kaijzel, E., Koolwijk, P., Van Erck, M., Van Hinsbergh, V., De Maat, M.: Molecular weight fibrinogen variants determine angiogenesis rate in a fibrin matrix in vitro and in vivo. J. Thromb. Haemostasis 4(9), 1975-1981 (2006)

19. Koolwijk, P., van Erck, M., de Vree, W., Vermeer, M., Weich, H., Hanemaaijer, R., van Hinsbergh, V.: Cooperative effect of TNFalpha, bFGF, and VEGF on the formation of tubular structures of human microvascular endothelial cells in a fibrin matrix. Role of urokinase activity. J. Cell Biol. 132(6), 1177-88 (1996)

20. Kroon, M., Koolwijk, P., Van Goor, H., Weidle, U., Collen, A., Van Der Pluijm, G., van Hinsbergh, V.: Role and localization of urokinase receptor in the formation of new microvascular structures in fibrin matrices. Am. J. Pathol. 154(6), 1731 (1999)

21. Liu, G., Qutub, A., Vempati, P., Mac Gabhann, F., Popel, A.: Module-based multiscale simulation of angiogenesis in skeletal muscle. Theor. Biol. Med. Modell. 8(1), 6 (2011). DOI $10.1186 / 1742-4682-8-6$

22. Manoussaki, D., Lubkin, S., Vemon, R., Murray, J.: A mechanical model for the formation of vascular networks in vitro. Acta Biotheor. 44(3), 271-282 (1996)

23. McDougall, S., Anderson, A., Chaplain, M.: Mathematical modelling of dynamic adaptive tumour-induced angiogenesis: clinical implications and therapeutic targeting strategies. J. Theor. Biol. 241(3), 564-89 (2006). DOI 10.1016/j.jtbi.2005.12.022

24. Merks, R., Brodsky, S.V., Goligorksy, M.S., Newman, S.A., Glazier, J.A.: Cell elongation is key to in silico replication of in vitro vasculogenesis and subsequent remodeling. Dev. Biol. 289, 44-54 (2006)

25. Merks, R., Glazier, J.: A cell-centered approach to developmental biology. Phys. A 352(1), $113-130$ (2005)

26. Merks, R., Glazier, J.: Dynamic mechanisms of blood vessel growth. Nonlinearity 19(1) (2006)

27. Merks, R., Koolwijk, P.: Modeling Morphogenesis in silico and in vitro: Towards Quantitative, Predictive, Cell-based Modeling. Math. Model. Nat. Phenom. 4(4), 149-171 (2009). DOI $10.1051 / \mathrm{mmnp} / 20094406$

28. Merks, R., Newman, S., Glazier, J.: Cell-oriented modeling of in vitro capillary development. In: P. Sloot, B. Chopard, A. Hoekstra (eds.) Cellular Automata, Lecture Notes in Computer Science, vol. 3305, pp. 425-434. Springer Berlin / Heidelberg (2004)

29. Merks, R., Perryn, E., Shirinifard, A., Glazier, J.: Contact-inhibited chemotaxis in de novo and sprouting blood-vessel growth. PLoS Comput. Biol. 4(9), e1000,163 (2008)

30. Montesano, R., Pepper, M., Möhle-Steinlein, U., Risau, W., Wagner, E., Orci, L.: Increased proteolytic activity is responsible for the aberrant morphogenetic behavior of endothelial cells expressing the middle T oncogene. Cell 62(3), 435-445 (1990)

31. Namy, P., Ohayon, J., Tracqui, P.: Critical conditions for pattern formation and in vitro tubulogenesis driven by cellular traction fields. J. Theor. Biol. 227, 103-120 (2004)

32. Qutub, A., Liu, G., Vempati, P., Popel, A.: Integration of angiogenesis modules at multiple scales: from molecular to tissue. In: Pacific Symposium on Biocomputing. Pacific Symposium on Biocomputing, p. 316. NIH Public Access (2009)

33. Qutub, A., Mac Gabhann, F., Karagiannis, E., Vempati, P., Popel, A.: Multiscale Models of Angiogenesis. IEEE Eng. Med. Biol. 28(2), 14-31 (2009)

34. Qutub, A., Popel, A.: Elongation, proliferation \& migration differentiate endothelial cell phenotypes and determine capillary sprouting. BMC Syst. Biol. 3(1), 13 (2009)

35. Savill, N.: Modelling morphogenesis: from single cells to crawling slugs. J. Theor. Biol. 184(3), 229-235 (1996). DOI 10.1006/jtbi.1996.0237

36. Serini, G., Ambrosi, D., Giraudo, E., Gamba, A., Preziosi, L., Bussolino, F.: Modeling the early stages of vascular network assembly. EMBO J. 22, 1771-9 (2003) 
37. Shirinifard, A., Gens, J., Zaitlen, B., Popawski, N., Swat, M., Glazier, J.: 3D multicell simulation of tumor growth and angiogenesis. PLoS one 4(10) (2009). DOI 10.1371/journal.pone.0007190

38. Sholley, M., Ferguson, G., Seibel, H., Montour, J., Wilson, J.: Mechanisms of neovascularization. Vascular sprouting can occur without proliferation of endothelial cells. Lab. Invest. 51(6), 624 (1984)

39. Swat, M.H., Hester, S.D., Heiland, R.W., Zaitlen, B.L., Glazier, J.A., Shirinifard, A.: CompuCell3D Manual and Tutorial Version 3.6.0 (2011)

40. Szabó, A., Czirók, A.: The Role of Cell-Cell Adhesion in the Formation of Multicellular Sprouts. Math. Model. Nat. Phenom. 5(1) (2010). DOI 10.1051/mmnp/20105105

41. Szabó, A., Mehes, E., Kosa, E., Czirók, A.: Multicellular sprouting in vitro. Biophys. J. 95(6), 2702-2710 (2008). DOI 10.1529/biophysj.108.129668

42. Szabó, A., Perryn, E., Czirok, A.: Network formation of tissue cells via preferential attraction to elongated structures. Phys. Rev. Lett. 98(3), 038,102 (2007)

43. Tosin, a., Ambrosi, D., Preziosi, L.: Mechanics and chemotaxis in the morphogenesis of vascular networks. Bull. Math. Biol. 68(7), 1819-36 (2006). DOI 10.1007/s11538-006-9071-2

44. Vailhé, B., Ronot, X., Tracqui, P., Usson, Y., Tranqui, L.: In vitro angiogenesis is modulated by the mechanical properties of fibrin gels and is related to alpha(v)beta3 integrin localization. In Vitro Cell. Dev.-An. 33(10), 763-73 (1997). URL http://www.ncbi.nlm.nih.gov/pubmed/9466681

45. Van Hinsbergh, V., Koolwijk, P.: Endothelial sprouting and angiogenesis: matrix metalloproteinases in the lead. Cardiovasc. Res. 78(2), 203 (2008)

46. Vernon, R., Angello, J., Iruela-Arispe, M., Lane, T., Sage, E.: Reorganization of basement membrane matrices by cellular traction promotes the formation of cellular networks in vitro. Lab. Invest. 66(5), 536 (1992)

47. Weijers, E., van Wijhe, M., Joosten, L., Horrevoets, A., de Maat, M., van Hinsbergh, V., Koolwijk, P.: Molecular weight fibrinogen variants alter gene expression and functional characteristics of human endothelial cells. J. Thromb. Haemostasis 8(12), 2800-2809 (2010) 